\title{
Visualizing lone pairs in compounds containing heavier congeners of the carbon and nitrogen group elements
}

\author{
RAM SESHADRI \\ Solid State and Structural Chemistry Unit, Indian Institute of Science, \\ Bangalore 560 012, India \\ e-mail: seshadri@sscu.iisc.ernet.in
}

\begin{abstract}
In this mini-review, I discuss some recent work on the stereochemistry and bonding of lone pairs of electrons in divalent compounds of the heavier carbon group elements $\left(\mathrm{Sn}^{\mathrm{HI}}, \mathrm{Pb}^{\mathrm{II}}\right)$ and in trivalent compounds of the heavier nitrogen group elements $\left(\mathrm{Bi}^{\mathrm{III}}\right)$. Recently developed methods that permit the real-space visualization of bonding patterns on the basis of density functional calculations of electronic structure, reveal details of the nature of $s$ electron lone pairs in compounds of the heavier main group elements - their stereochemistry and their inertness (or lack thereof). An examination of tetragonal P4/nmm SnO, $\alpha$-PbO and BiOF, and cubic $F m \overline{3} m$ PbS provides a segue into perovskite phases of technological significance, including ferroelectric $\mathrm{PbTiO}_{3}$ and antiferroelectric/piezoelectric $\mathrm{PbZrO}_{3}$, in both of which the lone pairs on $\mathrm{Pb}$ atoms play a pivotal rôle.
\end{abstract}

Keywords. Main group elements; lone pairs; stereochemistry; ferroelectricity.

\section{Introduction}

The valence shell electron pair repulsion (VSEPR) theory, first suggested by Sidgwick and Powell ${ }^{1}$, to be refined later by Gillespie and Nyholm ${ }^{2}$, is the stuff of high school chemistry. The sequence of decreasing repulsion, lone pair-lone pair > lone pair-bond pair > bond pair-bond pair, is a familiar thumb rule that permits thousands of school and college students to hazard the geometry of molecules such as $\mathrm{XeO}_{3}$. While it is usual to assume that these thumb rules have a basis in hard quantum chemical theory, in reality, even the experimental structures of these simple molecules are sometimes a matter of controversy.

Regarding the disposition of lone pairs in extended solids, Sten Andersson and coworkers have in a series of papers ${ }^{3}$, come up with modifications of VSEPR theory that permit a better prediction of coordination polyhedra. The new rules are described in the magnum opus of Hyde and Andersson ${ }^{4}$ and include, crucially, the definition of a typical atom-lone pair distance, and the notion that in the space of the unit cell, the lone pair occupies as much volume as an oxide or fluoride anion.

At the same time, the question of what constitutes a chemical bond has received renewed attention, with key contributions from Richard Bader in his Atoms In Molecules approach $^{5}$. The electron localization function (ELF), of Becke and Edgecombe ${ }^{6}$ measures the extent of the Pauli repulsion, i.e. the conditional probability of finding an electron in the vicinity of another electron of like spin, and has seen much use in the demarcation of the space of a molecule or solid into regions which are bonding, nonbonding or antibonding. Such analysis has been further extended by the work of Silvi and 
Savin ${ }^{7}$ who in effect, have coupled the topological approach of Bader with the evaluation of ELF.

In this contribution, I present some recent attempts to understand structure in compounds that contain stereochemically active lone pairs of $s$ electrons. The basis for this is density functional calculations of the electronic structure followed by ELF visualizations. It is found that the anion and the degree of covalency are important for the manner in which the lone pairs dispose themselves. These studies also throw light on socalled 'inert pair' effects ${ }^{8}$ and support the view that these effects have rather complex origins that necessitate an analysis beyond the usual atomistic interpretations.

The outline of the article is as follows. First, I perform density functional electronic structure calculations as implemented in the scalar relativistic linear muffin-tin orbital method $^{9}$, on some typical lone pair compounds including $\mathrm{SnO}, \propto \mathrm{PbO}$ and BiOF. This permits plots of the ELF to be made, which when plotted in the real space of the crystal structure, permits the lone pair to be visualized, even in structures where it does not form a lobe - an example of such a structure being cubic PbS. I then consider the structures of more complex materials, namely the perovskites $\mathrm{PbTiO}_{3}$ and $\mathrm{PbZrO}_{3}$, and inquire into the rôle of the lone pair in driving (respectively) the ferroelectric and antiferroelectric phase transitions.

\section{SnO, $\alpha-P b O$, BiOF, PbS}

$\mathrm{SnO}$ and $\propto \mathrm{PbO}$ adopt a tetragonal structure ${ }^{4}$ with double slabs of the cation which sandwich $\mathrm{O}$, each four-coordinate with the other and stacked along the $c$ axis. In BiOF, the $\mathrm{BiO}$ network forms similar double slabs except that there are $\mathrm{F}^{-}$ions arranged in the interslab space above and below the cations. Interaction across the slabs is important for the semi-metallicity of $\mathrm{SnO}$ and for the transformation of $\alpha \mathrm{PbO}$ to a black $\gamma$ phase under pressure, before it transforms to the red massicot phase ${ }^{10}$

The structures of these three compounds are respectively displayed in figures 1a through $1 \mathrm{c}$. The large gray spheres are the cations and the orange spheres are $\mathrm{O}$. In $\mathrm{BiOF}$, fluorines have been represented by cyan spheres in the interslab region. In the panels of figure 1 , the beige lobes in the vicinity of the cations in the inter-slab space are the lone pairs, visualized as isosurfaces with an ELF value of $0 \cdot 85$, corresponding to rather strong localization. In a plane midway through the structure, the ELF is presented as a map, following the colour coding indicated in the bar at the bottom. Regions of poor localization (nearly free electrons) are indicated by deep blue and regions of strong localization (corresponding to an ELF value of one) are indicated by white.

First, this figure illustrates that is is now routinely possible to visualize in the real space of the crystal structure, the disposition of lone pairs from reliable first-principles calculations. This obviates the need to position lone pairs by guess work. Second, rather subtle features emerge, that cannot possibly be guessed. For example, one observes that there is some interaction between the lone pairs and $\mathrm{O}$ within the slabs revealed (particularly for $\mathrm{SnO}$ ) as a lightening in the colour in the appropriate region of the map. Additionally, the lone pairs in $\mathrm{PbO}$ are more compact when compared with $\mathrm{SnO}$, in keeping with the empirical findings of Andersson and coworkers ${ }^{4}$, that the lone pairatom distance is larger for $\mathrm{Sn}^{\mathrm{II}}$ than it is for $\mathrm{Pb}^{\mathrm{II}}$. In conjunction with the fact that the $\mathrm{Sn}-$ $\mathrm{O}$ system is much more covalent ${ }^{10}$, the more compact lone pair on $\mathrm{Pb}$ reveals the reluctance of $\mathrm{Pb}^{\mathrm{II}}$ toward oxidation to $\mathrm{Pb}^{\mathrm{IV}}$. This in fact is a manifestation of the inert pair effect - the propensity of the post-lanthanoid main-group elements to retain a valence 


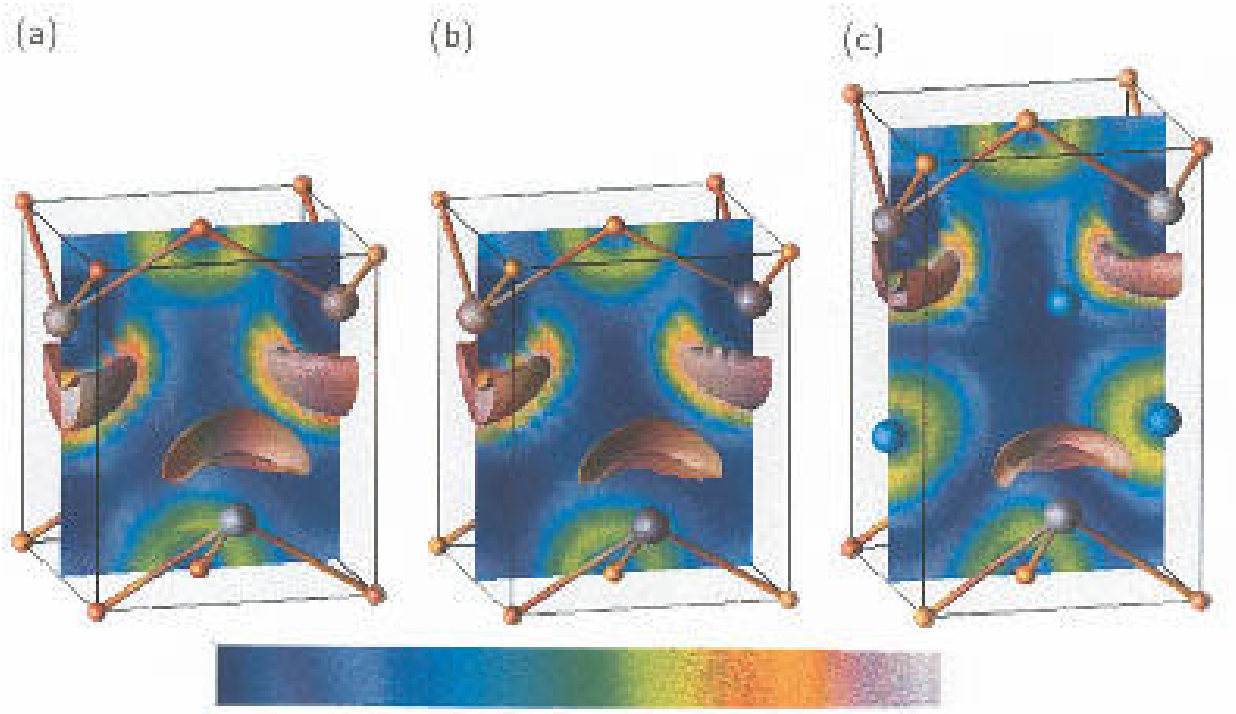

Figure 1. Crystal structures of (a) $\mathrm{SnO}$, (b) $\alpha-\mathrm{PbO}$, and (c) BiOF. Gray spheres are cations, orange $\mathrm{O}$ and cyan $\mathrm{F}$. In the space of the structures, isosurfaces of the electron localization function (ELF) with a value of 0.85 reveal the lone pairs as lobes near the cations. The map halfway through the structure is the ELF, represented by colour coding following the bar at the base of the figure. The deep blue end corresponds to poor localization and the white end to strong localization.

that is less than the group valence by two electrons. It is known that such a reluctance to attain the group valence cannot be explained merely from trends of ionization energies ${ }^{8}$. Electron localization in BiOF visualized in figure 1c suggests a much more ionic system with little or no interaction between $\mathrm{F}$ and the lone pairs, and this is confirmed by an analysis of the densities of state.

The lone pairs are expected to have primarily $5 s(\mathrm{Sn})$ or $6 s$ character $(\mathrm{Pb}, \mathrm{Bi})$. If they are to form lobes, there must be some $p$ admixture. The question is whether the $p$ character arises from mixing $s$ and $p$ on the cation or whether it is the effect of covalency, arising from cation $s$, anion $p$ mixing. Watson and Parker ${ }^{11}$ have recently suggested that it is due to $p$ character from the anions that lone pairs are allowed to form lobe-shaped structures. The anion is clearly implicated since in a homologous series of compounds, changing the anion often greatly affects the stereochemistry of the lone pair. In the case of $\mathrm{PbO}$, the density of $\mathrm{Pb} s$ and $\mathrm{Pb} p$ electronic states are well-separated (figure $2 \mathrm{a}$ ) in energy, while there seem to be $p$ states of $\mathrm{O}$ in the region of energy where $s$ states of $\mathrm{Pb}$ are found (figure 2b). Crystal Orbital Hamiltonian Populations (COHPs) permit the decomposition of densities of state into specific pairwise interactions between atoms ${ }^{12}$. Positive COHPs correspond to bonding interactions and negative to antibonding. The $\mathrm{Pb}-$ $\mathrm{O}$ COHP for $\propto \mathrm{PbO}$ is plotted in figure 2c. It is seen that the coincidence of $\mathrm{O} p$ and $\mathrm{Pb} s$ densities of state at an energy of $-8 \mathrm{eV}$ (referenced to the top of the valence band) does indeed correspond to strongly covalent interactions.

If lone pair $s$ must overlap with anion $p$ levels in order to attain the typical lobe shape, then conversely, in a situation where there is no such overlap, or when the overlap is 

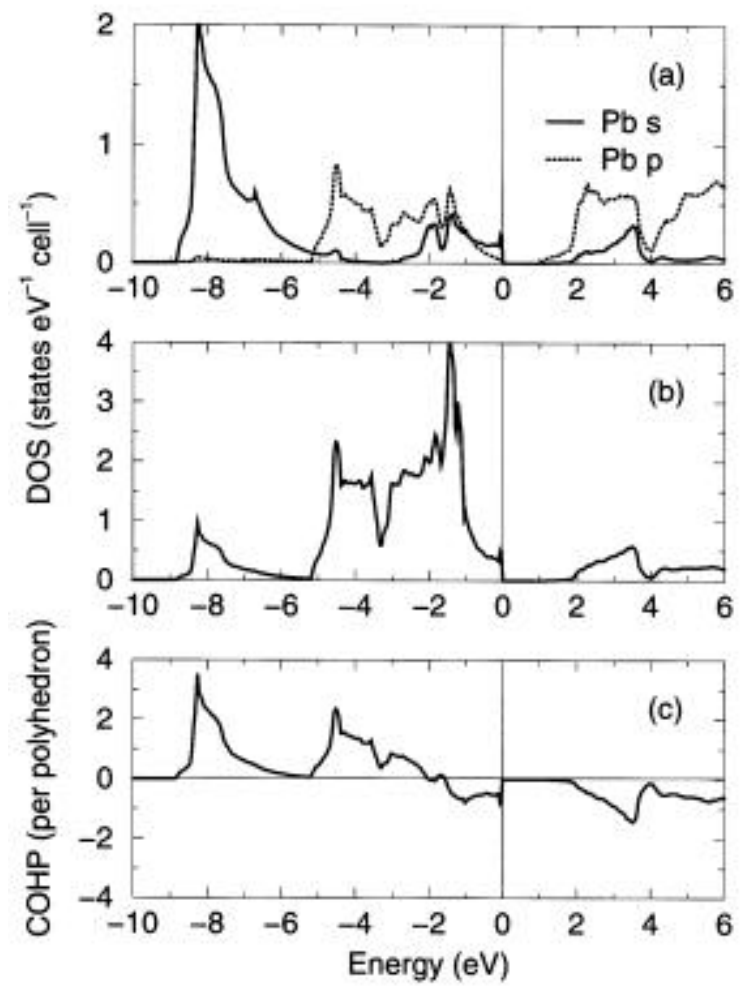

Figure 2. (a) Orbital projected density of $\mathrm{Pb} s$ and $p$ states in $\alpha-\mathrm{PbO}$. (b) Density of $\mathrm{O} p$ states in $\alpha-\mathrm{PbO}$. (c) Crystal orbital Hamiltonian population (COHP) of $\mathrm{Pb}-\mathrm{O}$ bonding in $\alpha-\mathrm{PbO}$. Positive values correspond to bonding interactions and negative values to antibonding interactions. The origin on the energy axis is the top of the valence band.

weak, the lone pair could retain its $s$ character and remain spherical. PbS is a strongly covalent system in terms of $\mathrm{Pb}-p \mathrm{~S}-p$ overlap, but (i) the $p$ levels of $\mathrm{S}$ are not as deep in energy as $\mathrm{O} p$ to bond strongly with $\mathrm{Pb} s$ and (ii) the intrinsically longer $\mathrm{Pb}-\mathrm{S}$ distance (due to larger atomic radii) may lessen overlap. The densities of state and the COHP of cubic $(\mathrm{NaCl}) \mathrm{PbS}$ are displayed in figure 3 to support this view. It is seen that while there is some overlap of $\mathrm{Pb} s$ with anion $p$ states, it is much weaker than what is seen in $\alpha \in \mathrm{PbO}$. One octant of the rock-salt structure of $\mathrm{PbS}$ is displayed in figure 4. The yellow spheres are $\mathrm{S}$ and the gray spheres $\mathrm{Pb}$. A map of the ELF is displayed at one end of the structure. In order to visualize the lone pairs around $\mathrm{Pb}$, it was necessary to select an isosurface with an ELF value of 0.6 signifying rather poor localization of the lone pair. At this value, spheres of localization around $\mathrm{S}$ atoms are also seen. As anticipated from the discussion, the lone pairs around $\mathrm{Pb}$ are nearly spherical in shape.

\section{3. $\mathrm{PbTiO}_{3}$}

On cooling below $766 \mathrm{~K}, \mathrm{PbTiO}_{3}$ undergoes a displacive phase transition (with a textbook soft mode) from an ideal cubic perovskite $(P m \overline{3} m)$ to a ferroelectric tetragonal 

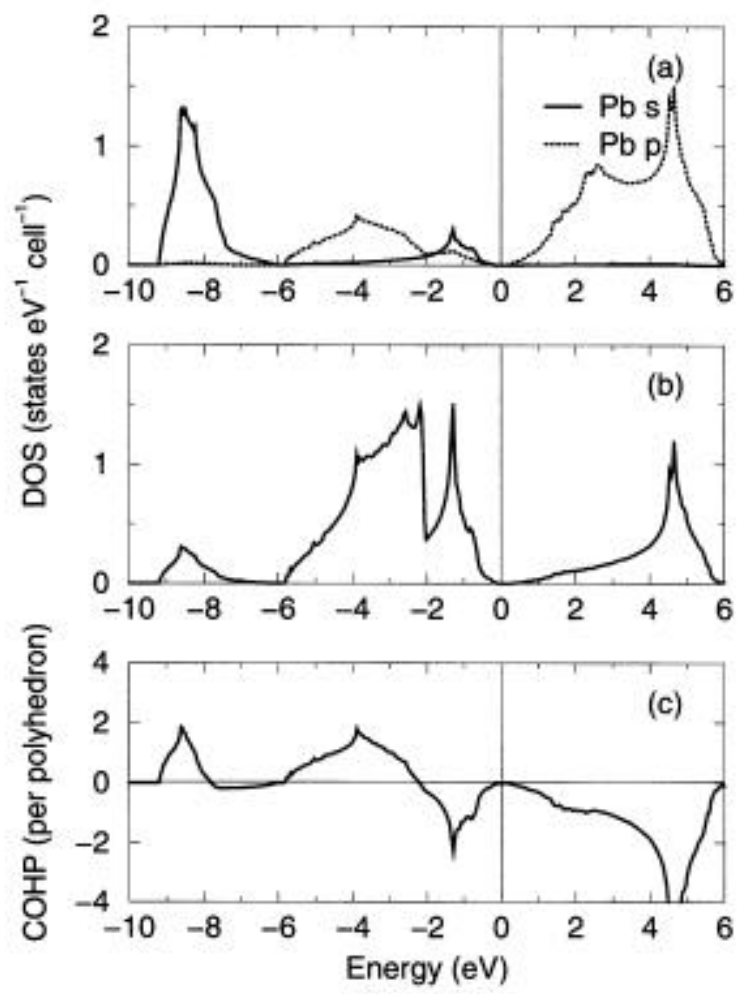

Figure 3. (a) Orbital projected density of $\mathrm{Pb} s$ and $p$ states in $\mathrm{PbS}$. (b) Density of $\mathrm{S}$ $p$ states in $\mathrm{PbS}$. (c) $\mathrm{COHP}$ of $\mathrm{Pb}-\mathrm{S}$ bonding in $\mathrm{PbS}$.

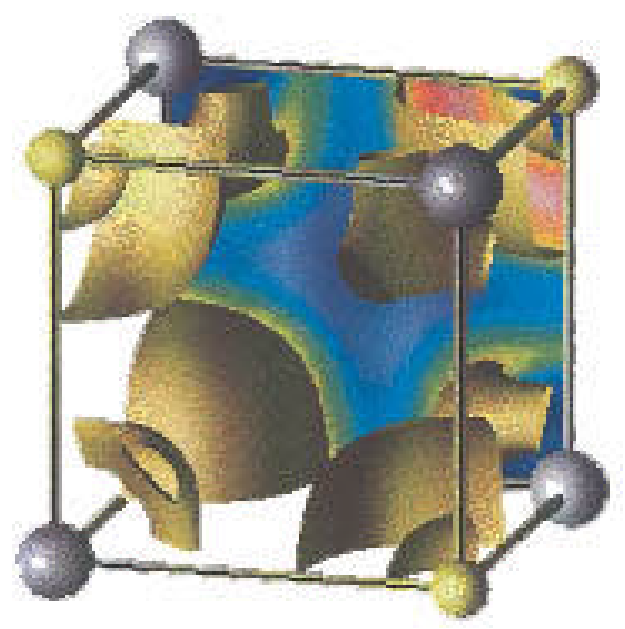

Figure 4. One octant of the rock salt structure of $\mathrm{PbS}$. Gray spheres are $\mathrm{Pb}$ and yellow spheres are S. A map of the ELF is displayed at one end of the octant. An ELF isosurface corresponding to a value of 0.6 is displayed, and indicates localization around both the cation and the anion. 
perovskite with the space group $\mathrm{P}_{4} \mathrm{~mm}^{13}$. There are two aspects of the $\mathrm{PbTiO}_{3}$ structure that need to be be considered. The first is the Goldschmidt tolerance factor which is calculated (in the cubic phase) to be about 1.03. This means that the distance in the cubic phase between $\mathrm{Pb}$ and $\mathrm{O}$ is a little too small, and one expects a tendency for the structure to undergo a distortion that would in effect permit a larger volume for $\mathrm{Pb}$. Secondly, $d^{0}$ transition metal ions (particularly $\mathrm{Ti}^{\mathrm{IV}}, \mathrm{V}^{\mathrm{V}}$ and $\mathrm{Cr}^{\mathrm{VI}}$ ) display a strong tendency to socalled second order Jahn-Teller distortions ${ }^{14}$ that result in off-centric coordination polyhedra around these ions. These two factors cooperate in obtaining the rather high Curie temperature of $\mathrm{PbTiO}_{3}$. By the same token, the tolerance factor of $\mathrm{BaTiO}_{3}$ is 1.07, and one would perhaps expect it to possess an even higher Curie temperature. In fact, the Curie temperature (again going from a cubic to a tetragonal phase) of $\mathrm{BaTiO}_{3}$ is only $393 \mathrm{~K}^{13}$. The distinct differences between $\mathrm{PbTiO}_{3}$ and $\mathrm{BaTiO}_{3}$ have been suggested as arising from the involvement of $\mathrm{Pb}$ states in bonding near the Fermi energy ${ }^{15}$.

Figure 5 displays the structure of cubic $\mathrm{PbTiO}_{3}{ }^{16}$ with gray $\mathrm{Pb}$ atoms in the corners of the cube and octahedral Ti (black) in the centre. Midway through the structure, a map of the ELF (following the usual colour code) is displayed. As in the case of PbS, the lone pair (visualized at an ELF value of 0.65) forms a nearly spherical object around $\mathrm{Pb}$. In the tetragonal phase (two unit cells shown in figure 6) it is seen that the distortion of the $\mathrm{TiO}_{6}$ octahedra act in concert with the localization of the lone pairs on $\mathrm{Pb}$ into lobes (visualized at an ELF value of 0.7). The manner in which the lone pairs dispose themselves suggests a greater tendency to the ferroelectric distortion and a reason for the higher Curie temperature.

In figure 7( $\mathrm{a}$ and $\mathrm{b}) \mathrm{I}$ compare the densities of state in cubic (displayed as solid lines) and tetragonal (displayed as dashed lines) $\mathrm{PbTiO}_{3}$, focusing on $\mathrm{Pb} s$ and $p$ states in panel (a) and $\mathrm{O} p$ states in panel (b). The separation of $\mathrm{Pb} s$ and $\mathrm{Pb} p$ in both the cubic and tetragonal phases is similar to what is seen in $\alpha-\mathrm{PbO}$, except perhaps that the $\mathrm{Pb} s$ states are less disperse. Reflecting the smaller dispersion of the $\mathrm{Pb} s$ states are correspondingly fewer $\mathrm{O} p$ states in this region of energy (figure $7 \mathrm{~b}$ ), $-8 \mathrm{eV}$ with reference to the Fermi energy. Once again, the COHPs (figure 7c) reveal details that are not evident in the DOS.

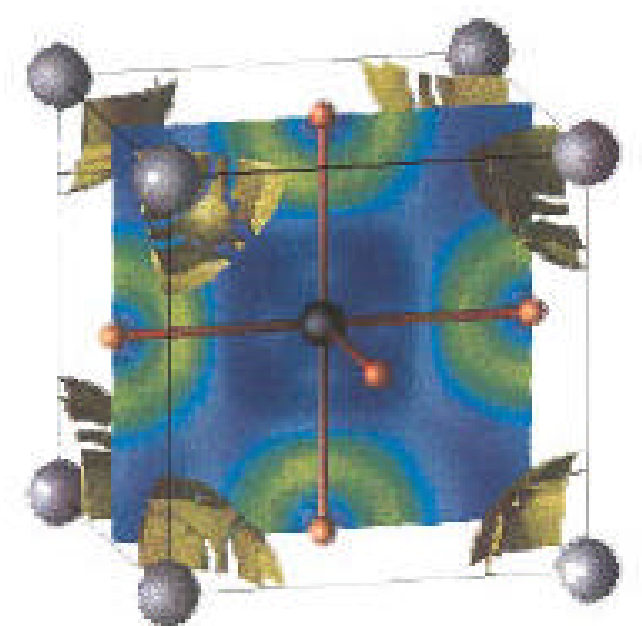

Figure 5. The structure of cubic $\mathrm{PbTiO}_{3}$. Gray spheres are $\mathrm{Pb}$, black Ti and orange O. The isosurface is at an ELF value of 0.65 and permits the nearly spherical (octahedral, in a stricter sense) lone pair to be visualized. 


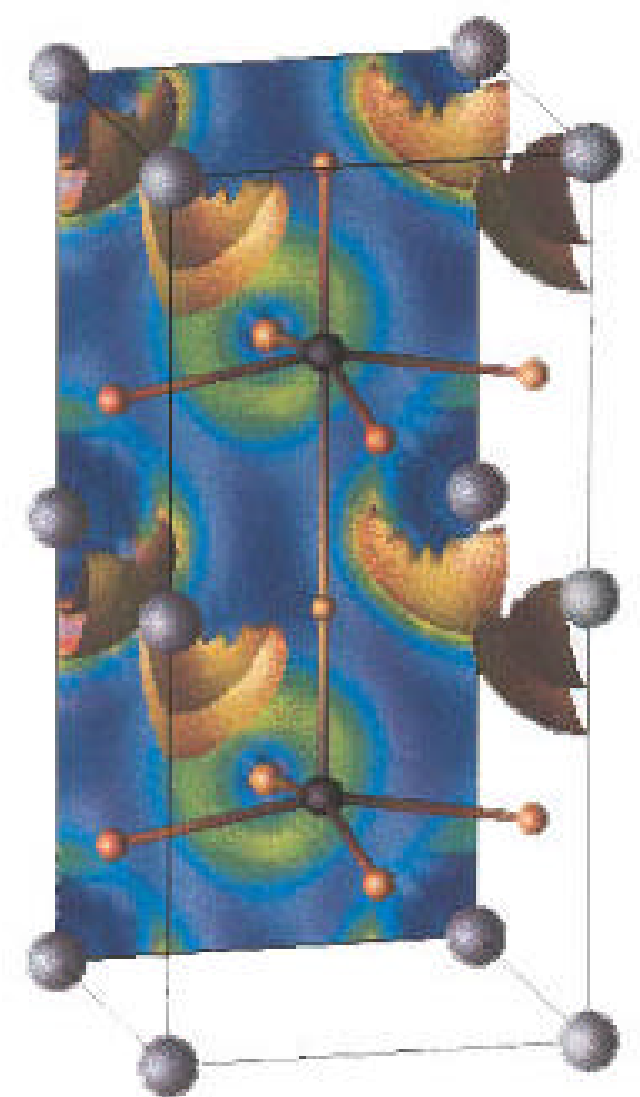

Figure 6. The structure of tetragonal, ferroelectric $\mathrm{PbTiO}_{3}$. Two unit cells are displayed. The lone pairs are visualized at an ELF isosurface of 0.7. If the frame of reference is the $\mathrm{O}$ in the plane perpendicular to $c$, both $\mathrm{Pb}$ and $\mathrm{Ti}$ are observed to be displaced in the same direction, due respectively to the lone pair and the second-order Jahn-Teller distortion.

It is seen that while $\mathrm{Pb} s-\mathrm{O} p$ bonding is not very strong, it is at least as important as the $\mathrm{Pb} p-\mathrm{O} p$ interaction. An interesting change across the phase transition is that the slightly stronger bonding between $\mathrm{Pb}$ and $\mathrm{O}$ in the tetragonal phase in the energy range corresponding to the lone pair, is compensated for by the development of antibonding states just below the Fermi energy. These antibonding states are missing in the cubic compound. Once again, the formation of the lone pair lobe is favoured by overlap between $\mathrm{Pb} s$ and $\mathrm{O} p$.

\section{4. $\mathrm{PbZrO}_{3}$}

$\mathrm{Zr}^{\mathrm{IV}}$ is significantly larger than $\mathrm{Ti}^{\mathrm{IV}}$ and as a result, the tolerance factor of $\mathrm{PbZrO}_{3}$ is about 0.97 . A tolerance factor that is less than 1 suggests that (unlike the case of $\mathrm{PbTiO}_{3}$ ) $\mathrm{Pb}$ is in a coordination polyhedron that is a little too large. If the octahedra tilt in a concerted manner, it is possible to slightly squeeze the polyhedron around $\mathrm{Pb}$ in order to achieve a more comfortable $\mathrm{Pb}-\mathrm{O}$ bond distance. Being a $d^{0}$ transition metal ion, $\mathrm{Zr}^{\mathrm{IV}}$ 

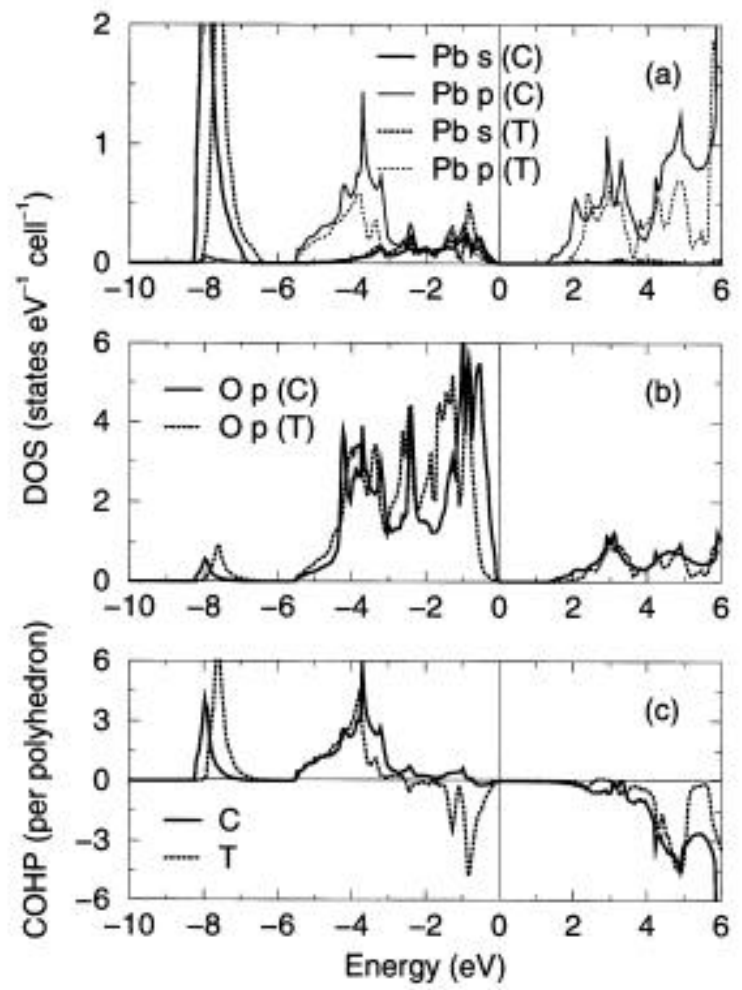

Figure 7. (a) $\mathrm{Pb} s$ and $p$ densities of state in the cubic (C) and tetragonal (T) structures of $\mathrm{PbTiO}_{3}$. (b) $\mathrm{O} p$ states in the cubic (C) and tetragonal (T) structures. $\mathrm{Pb}-\mathrm{O}$ COHP in the cubic (C) and tetragonal (T) structures.

would like to undergo an off-centric distortion within its oxygen octahedron though the tendency is reduced when compared with $\mathrm{Ti}^{\mathrm{IV}}{ }^{14}$. Finally, the $\mathrm{Pb}^{\mathrm{II}}$ ions on the A site of the structure possess a tendency to move away from the centre of their coordination polyhedron in order to permit the lone pair to take on the shape of a lobe. The two overwhelming driving forces for the phase transition in $\mathrm{PbZrO}_{3}$ at $503 \mathrm{~K}$ turn out to be the tendency of the $\mathrm{ZrO}_{6}$ octahedra to tilt in a cooperative manner (driven by a tolerance factor less than 1) and the tendency of the $\mathrm{Pb}$ atom lone pairs to localize into lobes. As a result of the $\mathrm{Pb}$ atoms displacement and the tilting not being cooperative, the resulting structure is antiferroelectric rather than ferroelectric ${ }^{13}$. Waghmare and Rabe ${ }^{17}$ have been able to reproduce the rather complex experimental structure in a completely ab-initio manner on the basis of density functional theory.

Electronic structure calculations using the crystal structure of orthorhombic $\mathrm{PbZrO}_{3}$ determined by Glazer and coworkers ${ }^{18}$ once again permit the disposition of $\mathrm{Pb}$ lone pairs within the space of the crystal structure to be visualized. Figure 8 displays the crystal structure with gray spheres representing $\mathrm{Pb}$ atoms, black $\mathrm{Zr}$ and orange $\mathrm{O}$. Isosurfaces of the ELF at a value of 0.7 permit reveal the lobe-like lone pairs possessed by the $\mathrm{Pb}$ atoms. These lone pairs are seen to arrange themselves in an antiferrodistortive manner resulting in the antiferroelectric properties of this orthorhombic phase. 


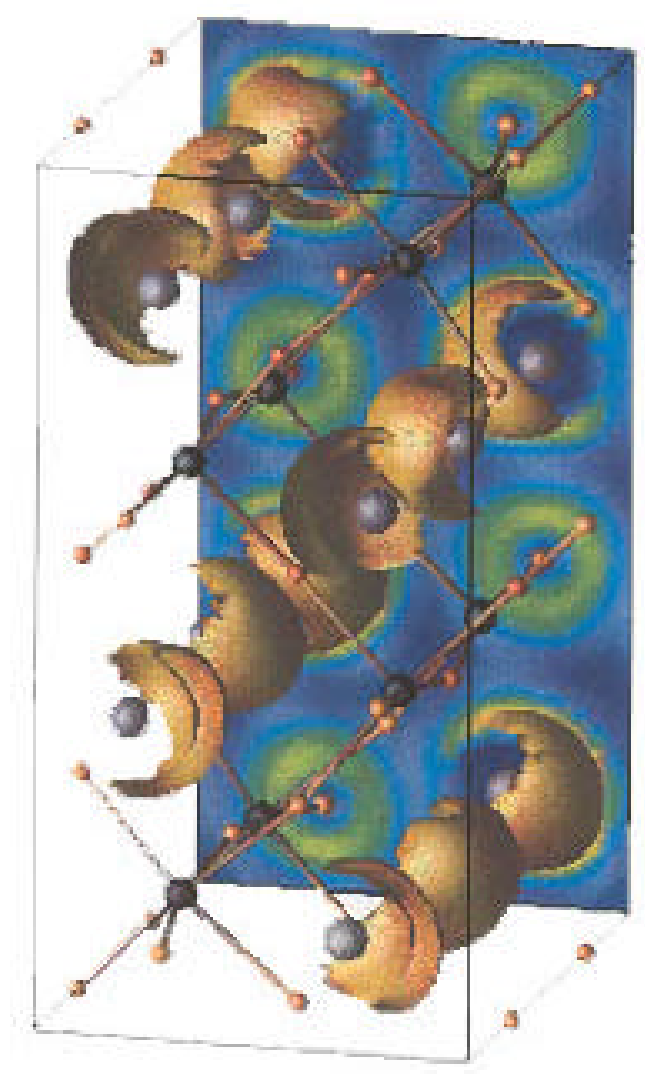

Figure 8. The structure of orthorhombic, antiferroelectric $\mathrm{PbZrO}_{3}$. The lone pairs are visualized at an ELF isosurface of 0.7. The displacements of the $\mathrm{Pb}$ atoms are such that their effects cancel out suggesting an antiferrodistortive structure.

\section{Prospects}

In brief, I have attempted to show that the stereochemistry of lone pairs in structures containing the heavier congeners of the carbon and nitrogen groups can be visualized on the basis of reliable density functional calculations of the electronic structure. Such visualization obviates the necessity to guess the role and disposition of lone pairs in determining structure. What is interesting is that even complex pyroelectric systems such as $\mathrm{PbTiO}_{3}$ and $\mathrm{PbZrO}_{3}$ turn out to be amenable to such analysis. The visualization of the lone pairs suggests reasons for the high Curie temperature of $\mathrm{PbTiO}_{3}$ and also suggests why $\mathrm{PbZrO}_{3}$ is antiferroelectric. Elsewhere, it has been suggested on the basis of such visualizations that lone pairs on $\mathrm{Pb}$ atoms are important in driving the cubicorthorhombic transition in $\mathrm{Pb}_{2} \mathrm{MgWO}_{6}{ }^{19}$. In the magnetic, possibly multiferroic $\mathrm{BiMnO}_{3}{ }^{20}$, the lone pairs on $\mathrm{Bi}$ are crucial for stabilizing ferromagnetism and in driving the off-centric distortion ${ }^{21}$.

An important aspect of the present work is the identification of conditions corresponding to when lone pairs localize and to when they remain spherical or nearly so. An interesting system in which these factors are manifest are the nine binary 
chalcogenides of the divalent carbon group elements, including GeS, GeSe ... SnSe, ... PbTe. If these are represented as a matrix of compositions, the upper left diagonal is unstable in the cubic rock-salt structure. We are currently examining how lone pairs help sort this structural field.

\section{Acknowledgements}

I thank O K Andersen and O Jepsen for generously making their LMTO codes available. I have greatly profited from discussions and continuing collaboration on the stereochemistry of lone pairs with G Baldinozzi, C Felser, N A Hill and U Waghmare. I am particularly grateful to J Gopalakrishnan for sharing his insights into crystal structures of compounds with $d^{0}$ elements, and to $\mathrm{N}$ A Hill for critically reading this manuscript. The Laboratoire SPMS and the École Centrale Paris are acknowledged for their kindness in inviting me for two months in the summer of 2001, when this article was written. This work has been supported by the Council of Scientific and Industrial Research, New Delhi.

\section{References}

1. Sidgwick N V and Powell H M 1940 Proc. R. Soc. London A176 153

2. Gillespie R J and Nyholm R S 1957 Q. Rev. Chem. Soc. 11339

3. Andersson S and Åström A 1972 Natl. Bur. Standards, 5th Mat. Symp.p 3; Galy J, Meunier G, Andersson S and Åström A 1975 J. Solid State Chem. 9 92; Andersson S 1979 Acta Crystallogr. B35 1321

4. Hyde B G and Andersson S 1989 Inorganic crystal structures (New York: John Wiley and Sons)

5. Bader R F W 1990 Atoms in molecules: A quantum theory (Oxford: University Press)

6. Becke A D and Edgecombe K E 1990 J. Chem. Phys. 9253

7. Silvi B and Savin A 1994 Nature (London) 371683

8. Huheey J E 1978 Inorganic chemistry, principles of structure and reactivity $2 \mathrm{nd}$ edn (New York: Harper \& Row)

9. Tank R W, Jepsen O, Burkhardt A and Andersen O K 1998 The Stuttgart TB-LMTO-ASA program (version 47), MPI für Festkörperforschung, Stuttgart, Germany; Jepsen O and Andersen O K 1995 Z. Phys. B97 35

10. Raulot J-M, Baldinozzi G, Seshadri R and Cortona P 2001 Solid State Sci. (submitted)

11. Watson G W and Parker S C 1999 J. Phys. Chem. B103 1258; Watson G W, Parker S C and Kresse G 1999 Phys. Rev. B59 8481

12. Dronskowski R and Blöchl P E 1993 J. Phys. Chem. 97 8617; Boucher F and Rousseau R 1998 Inorg. Chem. 372351

13. Lines M E and Glass A M 1977 Principles and applications of ferroelectrics and related materials (Oxford: Clarendon)

14. Bhuvanesh N S P and Gopalakrishnan J 1997 J. Mater. Chem. 72297

15. Cohen R E 1992 Nature (London) 358136

16. Glazer A M and Mabud S A 1978 Acta Crystallogr. B34 1065

17. Waghmare U V and Rabe K M 1997 Ferroelectrics 194135

18. Corker D L, Glazer A M, Dec J, Roleder K and Whatmore R W 1997 Acta Crystallogr. B53 135

19. Seshadri R, Baldinozzi G, Felser C and Tremel W 1999 J. Mater. Chem. 92463

20. Atou T, Chiba H, Ohoyama K, Yamaguchi Y and Syono Y 1999 J. Solid State Chem. 145639

21. Hill N A and Rabe K M 1999 Phys. Rev. $\mathbf{5 9 9}$ 8759; Seshadri R and Hill N A 2001 Chem. Mater. 132829 